\title{
Adapting the HLS-EU questionnaire for children aged 9 to 10: Exploring factorial validity
}

Torsten Michael Bollweg

TM Bollweg, O Okan, J Bröder, U Bauer, P Pinheiro

Faculty of Educational Science, Bielefeld University, Bielefeld, Germany

Contact: torsten.bollweg@uni-bielefeld.de

Background:

Health literacy (HL) in childhood has gained attention as an approach towards health promotion. Still, there is little evidence on HL of children, partly due to a lack of adequate assessment tools. This research gap was addressed by adapting and piloting the European Health Literacy Survey Questionnaire (HLS-EU-Q) for children aged 9-10.

Methods:

HLS-EU-Q items were evaluated and modified. The adapted self-report questionnaire, including 26 items assessing selfreported $\mathrm{HL}$, was used in a classroom survey among $\mathrm{n}=907$ children attending fourth grade of primary school in North Rhine-Westphalia, Germany. Exploratory and confirmatory factor analysis (EFA and CFA) were performed to assess factorial validity.

\section{Results:}

The theoretical factor structure of the HLS-EU model of HL (4 competence areas: accessing, understanding, appraising, applying health information) could not be replicated in EFA or CFA. EFA yielded a 6-factor model with one main factor (Eigenvalue $6.7 ; 25.6 \%$ explained variance), CFA showed strong correlations between the four latent factors, i.e. competence areas $(\mathrm{r}=.75-.92)$, and marginal to moderate factor loadings (.38$.64)$.

\section{Conclusions:}

This study is the first to adapt the HLS-EU-Q to measure selfreported $\mathrm{HL}$ of children aged 9 to 10 . The results on the factorial validity of this adapted version of the HLS-EU-Q put into question whether the four competence areas that are theoretically distinct in the underlying model can be appropriately assessed by the applied form of self-report assessment, as they are practically and statistically interrelated. Further research is necessary to verify these findings, and to investigate the potential of alternative approaches of conceptualizing and measuring $\mathrm{HL}$ of children. Eventually, this research aims to contribute to an advancement of this field of research and a solid evidence base on the HL of children that can inform political action and interventions to improve children's health through HL promotion. 